\title{
Application of Integrated Medical Care Model (Hospitalist) in Emergency Department and its Efficiency and Outcome: 4 years' Experience in Taiwan
}

Tzu-Chieh Weng ${ }^{1}$, Chien-Cheng Huang ${ }^{2-4}$, Chun-Cheng Zhang ${ }^{1}$, Hsin-Kai Huang ${ }^{1}$, Meng-Chieh Wu ${ }^{1}$, Chien-Chin Hsu ${ }^{2,5}$ and Kao-Chang Lin ${ }^{1,5,6 *}$

${ }^{1}$ Department of Holistic Care Unit, Chi-Mei Medical Center, Tainan, Taiwan

${ }^{2}$ Department of Emergency Medicine, Chi-Mei Medical Center, Tainan, Taiwan

${ }^{3}$ Bachelor Program of Senior Service, Southern Taiwan University of Science and Technology, Tainan, Taiwan

${ }^{4}$ Department of Environmental and Occupational Health, College of Medicine, National Cheng Kung University, Tainan, Taiwan

${ }^{5}$ Department of Biotechnology, Southern Taiwan University of Science and Technology, Tainan, Taiwan

${ }^{6}$ Department of Neurology, Chi Mei Medical Center, Tainan, Taiwan

\begin{abstract}
Background: The hospitalist model (HOS) setting at wards can improve quality of patient care and shorten length of stay, however, their efficacy at emergency department (ED) is still lacking in Taiwan.
\end{abstract}

Objective: To realize if HOS is working at ED in a designed academic care program.

Design/setting: Before-and-after intervention of HOS in a 1200-bed tertiary medical center in Taiwan.

Measurement: A three-shift duty with 8 internal subspecialists was adapted for their working schedule in 3:1:1 physicians round. From 2012 2016, 4 years data was collected retrospectively such as length of stay, over-waiting (>48 h) period, mortality, 6 and $24 \mathrm{~h}$ deterioration after hospitalization and satisfaction questionnaire were analyzed before and after HOS intervention was compared.

Results: There was no significant difference in patient number, age and sex before and after HOS program at ED. In total, 4 years data analyzed (520,409 ED visitors, 111,949 hospitalized) was shown that decline of waiting time, over-waiting $(>48 \mathrm{~h})$, mortality rate, post-hospitalized 6 and $24 \mathrm{~h}$ transferring to intensive care unit were statistically significant (ANOVA, $p \leqq 0.05$ individually). In average 55-60 patients per month discharged from holistic care unit without admission. Satisfactory questionnaire for pre-hospitalized care in disease explanation, service attitudes, symptom relief and overall impression about healthcare reached $90 \%$ in two times survey (427 and 459 sample sizes).

Conclusion: HOS at ED can lessen over-crowded conditions, shorten pre-hospitalized length, decline mortality, and improve quality of care and patient satisfaction. Although data comes from a single medical center, it still shows good efficiency and outcome since HOS implemented at emergency.

Keywords: Hospitalist program; Emergency department; Quality of care; Patient safety; Efficiency and outcome

\section{Introduction}

There are approximate 1200 emergency physicians responsible for the emergent care of totally 24 million people in Taiwan [1]. The increasing number of violent events at emergency department (ED) and work-related stress have greatly contributed to burnouts and shortage of emergent physicians $[2,3]$. Since it is much convenient for patients visiting ED with low-cost payments, the growing population has nearly overloaded all hospitals in Taiwan and increased the burdens of physician care $[4,5]$. These problems are urgent to be solved when hospitalization is warranted, no beds available at wards, and over-waiting time at ED. Prolonged ED waiting period is one of major causes of medical disputes $[4,6]$ in Taiwan. Such lawsuits frequently occurred when symptoms worse or mortality during pre-hospitalization or when management did not satisfy patient needs.

There are 19 medical centers in Taiwan, only two in Tainan city of southern Taiwan (Cheng Kung University Hospital and Chi Mei Medical Center-CMMC). CMMC [7] is responsible for at least 1.3 million people in Tainan area. It is a tertiary referring medical center serving approximately 12,000 patients who visited ED every month, with 2,300 patients (20\%) averagely required for hospitalization (Figure 1). Since the pre-hospitalized waiting time at ED was 6 to $96 \mathrm{~h}$ in average, it became worse in past years due to monopoly national health insurance in Taiwan $[5,8]$ with self-low-payment (patients paid less than $1 / 10$ and the others were paid by government). Herein the pivotal newly program of care model, hospitalist program-HOS, was launched by the hospital administrators to solve the over-crowded situations and cared pre-hospitalized patients in advance at ED.

The HOS was established in United Stated (US) in 1996 and the field of hospital medicine has grown rapidly worldwide recently. At present hospitalists on staffs reach 50,000 physicians, more than cardiologists, in almost all medical centers in US [9-12]. The advantages of HOS include a more rapid, effective and precise dealing with problems no matter in diagnoses, prescription of medications, therapeutic strategies or the communications with patients by sharing decision making with attending physicians rather than by residency in advance $[13,14]$. Several studies have reported that HOS model at wards provides shorter length of stay and improves quality of care [11,14-16]. From patient point of views,

${ }^{*}$ Corresponding author: Kao-Chang Lin, Department of Holistic Care Unit and Neurology, Chi-Mei Medical Center, 901 Zhonghua Road, Yongkang District, Tainan City 710, Taiwan, Tel: 886-6-281-2811; Fax: +886-6-281-6161; E-mail: gaujang@mail2000.com.tw

Received September 18, 2017; Accepted October 10, 2017; Published Octobe 17, 2017

Citation: Weng TC, Huang CC, Zhang CC, Huang HK, Wu MC, et al. (2017) Application of Integrated Medical Care Model (Hospitalist) in Emergency Department and its Efficiency and Outcome: 4 years' Experience in Taiwan. Health Care Current Reviews 5: 211. doi: 10.4172/2375-4273.1000211

Copyright: $\odot 2017$ Weng TC, et al. This is an open-access article distributed unde the terms of the Creative Commons Attribution License, which permits unrestricted use, distribution, and reproduction in any medium, provided the original author and source are credited. 
Citation: Weng TC, Huang CC, Zhang CC, Huang HK, Wu MC, et al. (2017) Application of Integrated Medical Care Model (Hospitalist) in Emergency Department and its Efficiency and Outcome: 4 years' Experience in Taiwan. Health Care Current Reviews 5: 211. doi: 10.4172/23754273.1000211



Figure 1: The ratio of visitors and pre-hospitalized waiting patients at emergency department (ED).

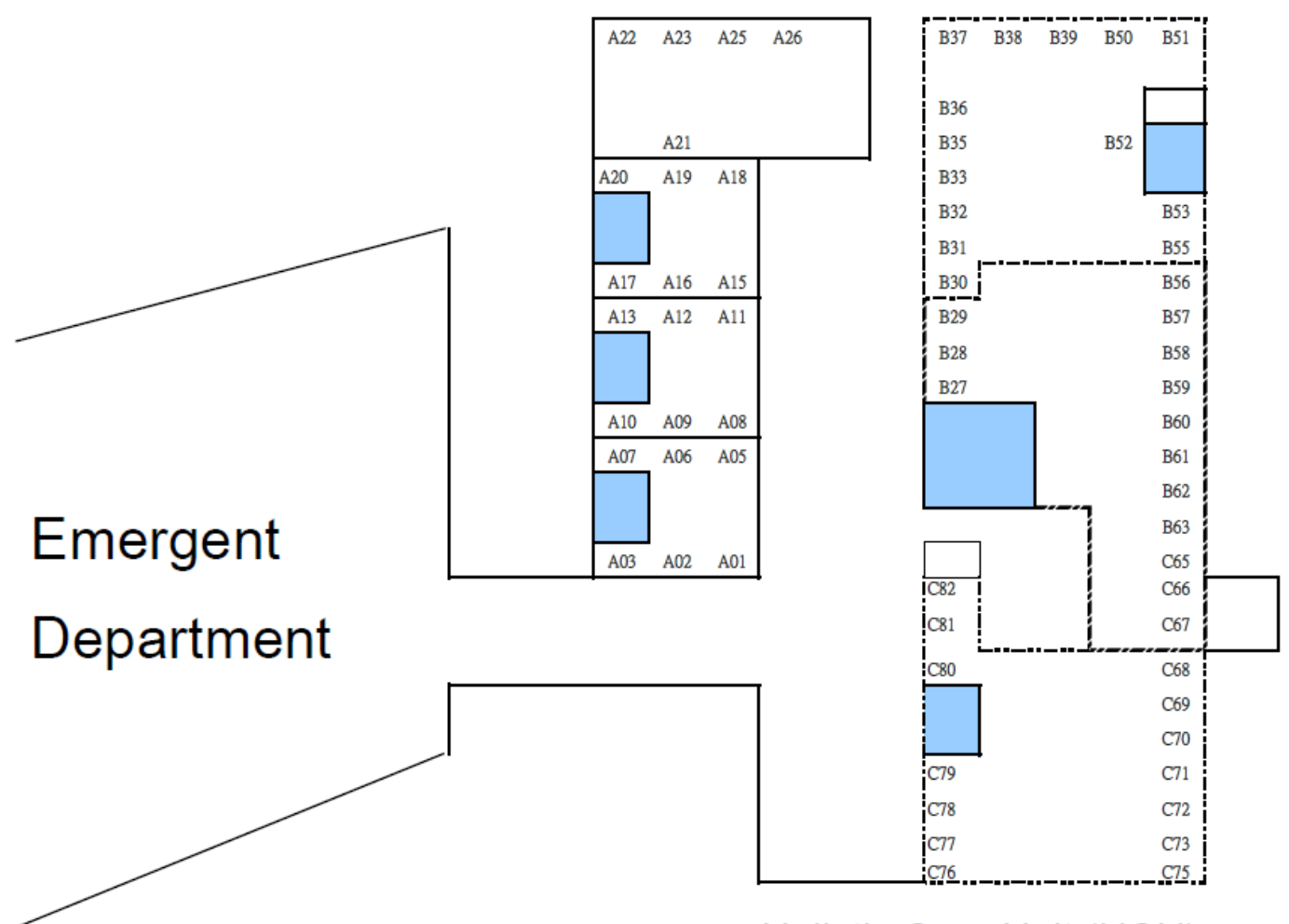

Holistic Care Unit (HCU)

Figure 2: The holistic care unit (HCU) nearby ED had 75-bed capacities for HOS program (blue color-6 nursing units).

HOS can provide more fast and accurate symptoms relief and shorter of hospital stay. Moreover, HOS has also been reported to decrease the medical expenditures and increase patient satisfaction [17-19]. The HOS in Taiwan healthcare system is just beginning since 2014 with payment incentives by experimental approach in selected 19 hospitals [20], its impact will affect the health policy undoubtedly, and the responsibility and burdens of care may shift from residency to attending physicians, no matter in hospitalists or subspecialists, in the future.

In Taiwan, the first trial of HOS in 2009, in National Taiwan University Hospital (NTUH) demonstrated shorter length of stay and saved medical cost about 1400 USD per person during hospitalization $[15,21]$. Since the overcrowded patients and limited available beds in many hospitals, the HOS care program at ED was established at CMMC since 2012, it was the first launch at ED in Taiwan, to take care of prehospitalized patients referred from emergency physicians. The hospitalists consisted of 8 internal subspecialists working together in Holistic Care Unit (HCU) at the observation room nearby ED. The throughput in HCU had 75 beds capacities for pre-hospitalized patient care (Figure 2 ) and mostly the over-waiting period ( $>48 \mathrm{~h}$ ) was around $6-8 \%$ before HOS established [22]. The initial preliminary 1 year result seemed to have improved quality of care and patient satisfaction, declined overcrowded conditions, mortality rate, and lessened medical disputes and lawsuits [22-24].

\section{Methods and Measures}

Members of the HCU included 8 subspecialist physicians with 1 
Citation: Weng TC, Huang CC, Zhang CC, Huang HK, Wu MC, et al. (2017) Application of Integrated Medical Care Model (Hospitalist) in Emergency Department and its Efficiency and Outcome: 4 years' Experience in Taiwan. Health Care Current Reviews 5: 211. doi: 10.4172/23754273.1000211

Page 3 of 6

neurologist, 1 geriatrist, 1 cardiologist, 1 pulmonologist, 1 nephrologist, 1 metabolic specialty, and 2 infectious physicians. The HCU team collaborated with emergency physicians for team discussion every morning within 365 days with efficient handovers on multiple comorbidities and critical ill patients. Using 3-color signals on the computer screen (green, yellow and red) to reveal the severity of illness so that physicians can seize immediately the severity of conditions before viewing patients. During waiting for admission, hospitalists with three-shift duty ( $3: 1: 1$ physicians round) will deal with all the problems of complaints as did inpatients at wards (pediatrics excluded). The consultations were made only for surgical interventions or devastating conditions of specific patients. The daytime three hospitalists (8:0016:00) routinely checked patients one by one with medications or coping strategy after visit, the night-shift (16:00-24:00) and midnightshift (24:00-08:00) dealt with unsolved conditions left. Within flexible $24 \mathrm{~h}$ care model, hospitalists on duty had less burdened and more responsibility for the safety and quality of patient care. Through good cooperation and experiences of HCU team with emergency physicians, the burnouts and resignations declined, and advanced symptom deterioration was prevented. If patients stabilized after aids by hospitalists, they were allowed to go home from HCU and deleted in waiting lists for admission. One case manager would follow postdischarge conditions of patients within $24 \mathrm{~h}$ by telephone interview and arranged back for clinic if necessary.

The data was collected from the hospital computer system at CMMC. We compared the ratio of visitors and pre-hospitalized waiting patients at ED between August 2012 and July 2016. Analysis of Variance (ANOVA) was used to compare the mean waiting hours, mortality rate in pre-hospitalized patients at $\mathrm{HCU}$, and $24 \mathrm{~h}$ transferring to intensive care unit after patients admission to wards before and after HOS implemented at ED. Patient satisfaction was analyzed using a questionnaire. We used the 5 -point Likert scale ( $1=$ very satisfied, $2=$ satisfied, $3=$ neutral, $4=$ dissatisfied, $5=$ =very dissatisfied) for analyzing the satisfaction of patients or caregivers. SPSS 20.0 was used for statistical analysis. The significance level was set at 0.05 (two tails).

\section{Ethics Statement}

This study was strictly conducted according to the Declaration of Helsinki and the requirements of the Institutional Review Board at CMMC. Informed consent for patients and caregivers was done and the questionnaires were anonymous.

\section{Results}

This study enrolled data from August 2012 to July 2016, before and after HOS implemented. The distributions of pre-hospitalized patients cared by hospitalists were gastro-enteric disease (10.27\%), urinary tract disease $(9.73 \%)$, respiratory disease $(8.1 \%)$, endocrine disorder $(7.05 \%)$ and cerebrovascular disease (6.19\%) and ratio of hospitalization within $24 \mathrm{~h}$ was $80 \%$. The critical patients waited for intensive care unit at ED were cardiovascular disorder (14.16\%), cerebrovascular disease $(11.80 \%)$, traumatic brain injury $(8.79 \%)$, respiratory disease $(6.3 \%)$ and bacteremia (4.84\%), and hospitalized rate within $24 \mathrm{~h}$ was $94 \%$. In total, 4 years data was analyzed (520,409 visitors at ED, 111,949 for hospitalization). The maximal decline of waiting time reached $21.75 \%$ at $3^{\text {rd }}$ year $(-3.71 \mathrm{~h}, \mathrm{p}=0.056)$ (Figure 3$)$, the over-waiting period $(>48$ h) declined peak by $47.3 \%$ at $3^{\text {rd }}$ year $(-4.64 \%, \mathrm{p}<0.01)$, the mortality declined by $62.5 \%$ peak at 2 nd-year $(-0.05 \%, \mathrm{p}<0.01)$ (Figure 4$)$, the 6 and $24 \mathrm{~h}$ symptoms deterioration after admission declined by $96.43 \%$ at $3^{\text {rd }}$ year (figure not shown) and $87.5 \%$ peak at $2^{\text {nd }}$ year (Figure 5) and shown statistically significant $(-0.27 \%$ vs. $-0.63 \%, \mathrm{p}<0.01$ individually). Pre-hospitalized patients discharged from HCU without admission was 2.21\% ( 55-60 patients per month). The rate of 3 day revisited ED was $1.98 \%$. The case manager traced patients after discharge with successful rate was mean $85.4 \%$ and backed to clinic was mean $81.3 \%$ by obedience. The medical lawsuits declined from 67 to 39 cases after HOS program implemented. Questionnaire for pre-hospitalized satisfaction about disease explanation, service attitudes, symptoms relief and overall impression in healthcare reached $90 \%$ in two investigations $\left(1^{\text {st }}\right.$ in 2013 and sample sizes $427 ; 2^{\text {nd }}$ in 2014 and sample sizes 459) (Figure 6).

\section{Discussion}

This 4 years pivotal approach of HOS care program revealed the benefits of decline overcrowded conditions at ED, shortened prehospitalized waiting time, reduced mortality rate, prevented further deterioration after hospitalization, improved patient satisfaction, and saved medical expenditure. It also developed good adherence and relationships between team members and patients after discharge. The mean waiting time and over-waiting period were declined with statistically significant before and after HOS with ANOVA statistics, in which the consistent result was revealed in $1^{\text {st }}$ year estimate. The efficient triage and experienced medical dealing by hospitalists might play some roles in declining waiting time for admission. It also saved medical costs for hospitalization. A total 16,667 USD/day was calculated in our patients (55-60 persons/month) without admission that saved the insurance cost indiscernibly. The over-waiting ( $>48 \mathrm{~h})$ at ED was decreased after HOS program from $7.71 \%$ to $3.07 \%$, yet, it still needed more efforts to solve this issue as speedy discharge process, available beds utility, or triaged to other hospitals. The decrease in mortality during pre-hospitalization at ED was more than half compared to previous HOS implemented (106 patients died in unpredictable

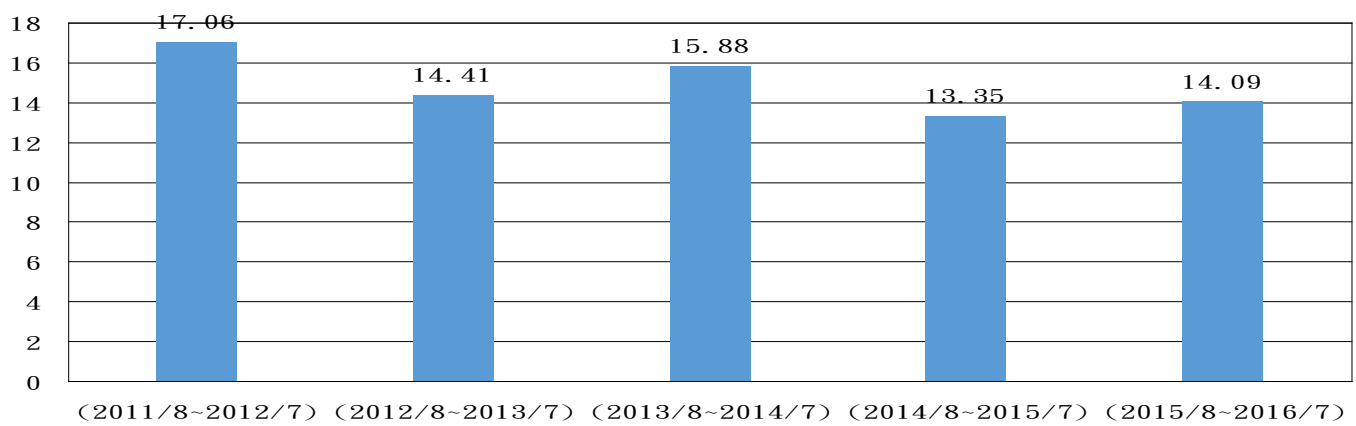

Figure 3: The mean waiting hours before and after hospitalist program (HOS) implemented at ED ( $p=0.056$, ANOVA). 
Citation: Weng TC, Huang CC, Zhang CC, Huang HK, Wu MC, et al. (2017) Application of Integrated Medical Care Model (Hospitalist) in Emergency Department and its Efficiency and Outcome: 4 years' Experience in Taiwan. Health Care Current Reviews 5: 211. doi: 10.4172/23754273.1000211

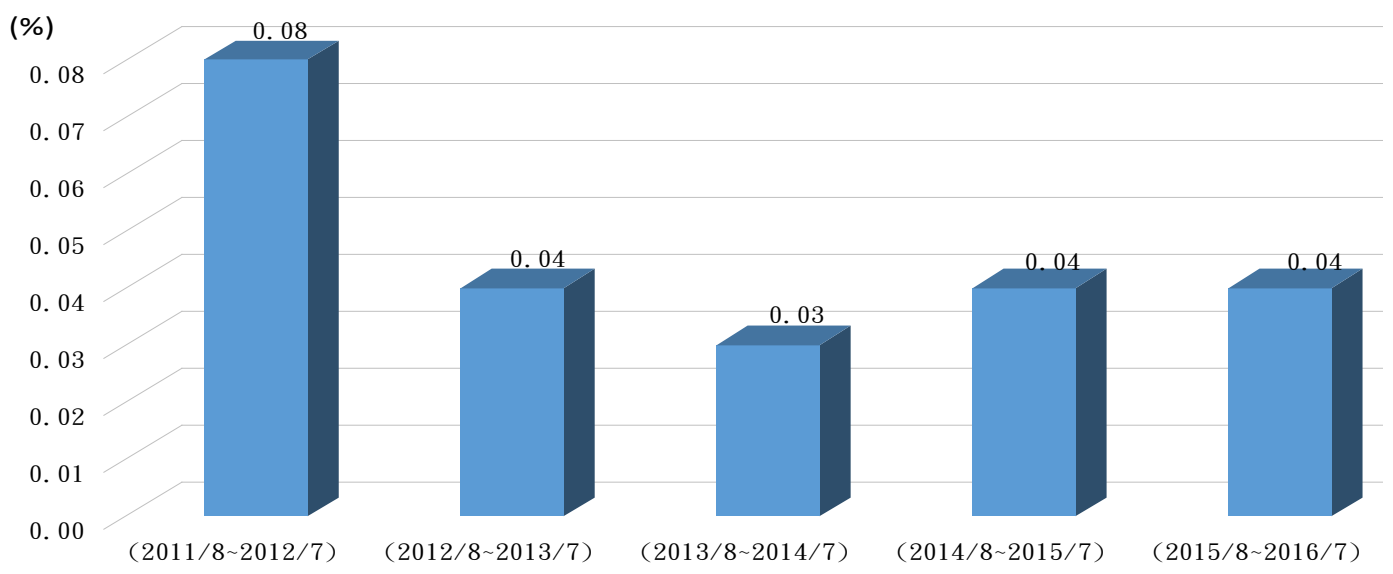

Figure 4: The mortality rate in pre-hospitalized patients at HCU before and after HOS program ( $p<0.01, A N O V A)$.

$(\%)$

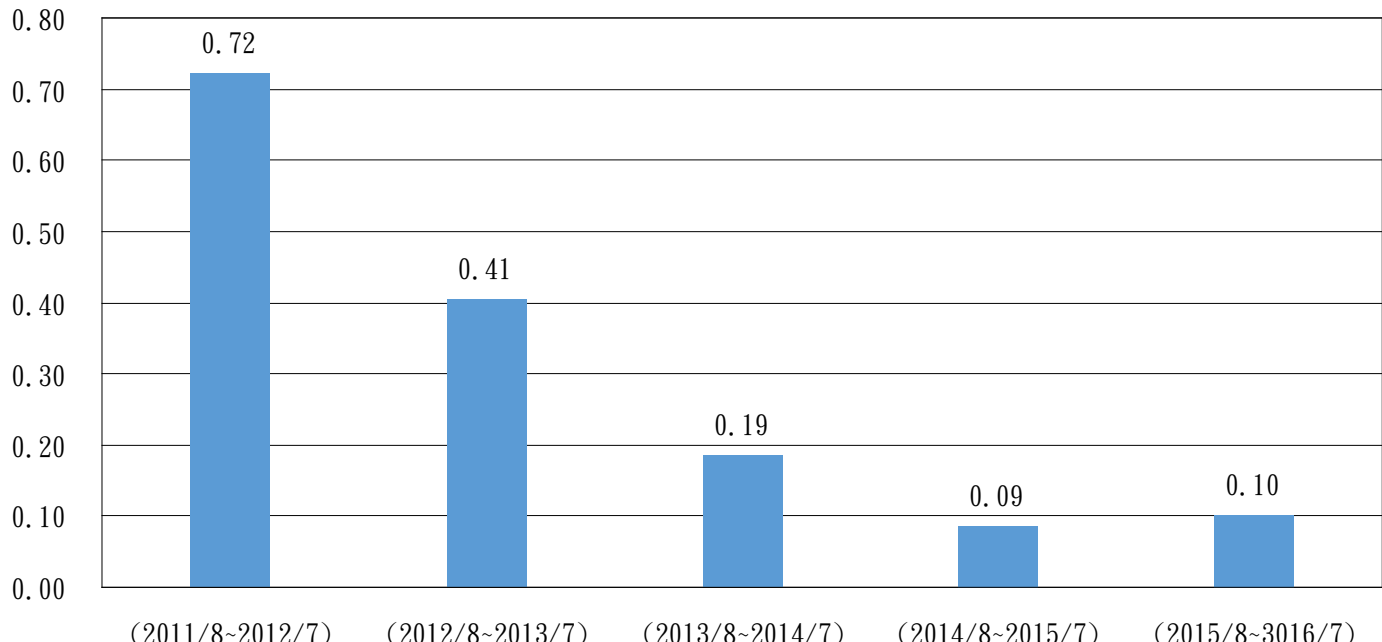

Figure 5: The $24 \mathrm{~h}$ transferring to intensive care unit after patients admission to wards before and after HOS program at ED ( $<<0.01, A N O V A)$.

situations and down to 39 patients in 2014, excluded Discharged against Medical Advise-DAMA). Arguments declined and satisfaction improved by regularly visited patients and managed all problems they complained. Hospitalists in HCU also unveiled subtle change that most emergency physicians easily neglected as pending hypoglycemia, unstable angina with normal cardiac enzyme and electrocardiography or stroke in evolution with normal computerized tomography, etc. The hospitalists had more confidence to predict deterioration and for ICU preparation in advance to prevent disputes in rush. From our analysis, the incidence of legal malpractice declined with statistically significant in our publication after HOS established [20]. The 6 and $24 \mathrm{~h}$ transferred to ICU after admission was also declined with statistically significant. This indicated that early bridging care and collaboration with team members could improve care quality and safety, decline mortality and length of stay and satisfaction to patients on waiting for admission at ED. It was once mentioned in literature [25].

A case manager followed up post-discharge patients within $24 \mathrm{~h}$ by telephone with successful rate from $1^{\text {st }}$ year of $75.7 \%$ to maximally
$89.4 \%$ (mean $85.4 \%$ ) and back to clinic follow-ups from $62.5 \%$ to $90 \%$ (mean 81.3\%) suggested that patients discharged from HCU had high adherence in our care program. Since patients backed home without hospitalization would be worried about symptoms flare, during which period a proper intervention by a case manager or specialist would be helpful to soothe anxiety mood and express concerns [26,27]. It was shown less disputes and declined in readmission possibilities by questionnaire.

The semi-structure Questionnaires regarding patient satisfaction of HOS model during pre-hospitalized period at ED were conducted in two different years and different patients. A 5-point Likert scale for rating the degree of satisfaction was used ( $1=$ very satisfied, $2=$ satisfied, $3=$ neutral, 4=dissatisfied, 5=very dissatisfied). Queries were used as "Did you satisfy about the working attitude in this care model of hospitalist setting unit so far?" or "Did you satisfy about your symptom relief by this hospitalists team care so far?" or "Did you overall satisfy about the care quality in this setting unit so far?" etc. The sample sizes were 427 and 459 respectively, and the satisfaction rate is higher to $90 \%$ 
Citation: Weng TC, Huang CC, Zhang CC, Huang HK, Wu MC, et al. (2017) Application of Integrated Medical Care Model (Hospitalist) in Emergency Department and its Efficiency and Outcome: 4 years' Experience in Taiwan. Health Care Current Reviews 5: 211. doi: 10.4172/23754273.1000211
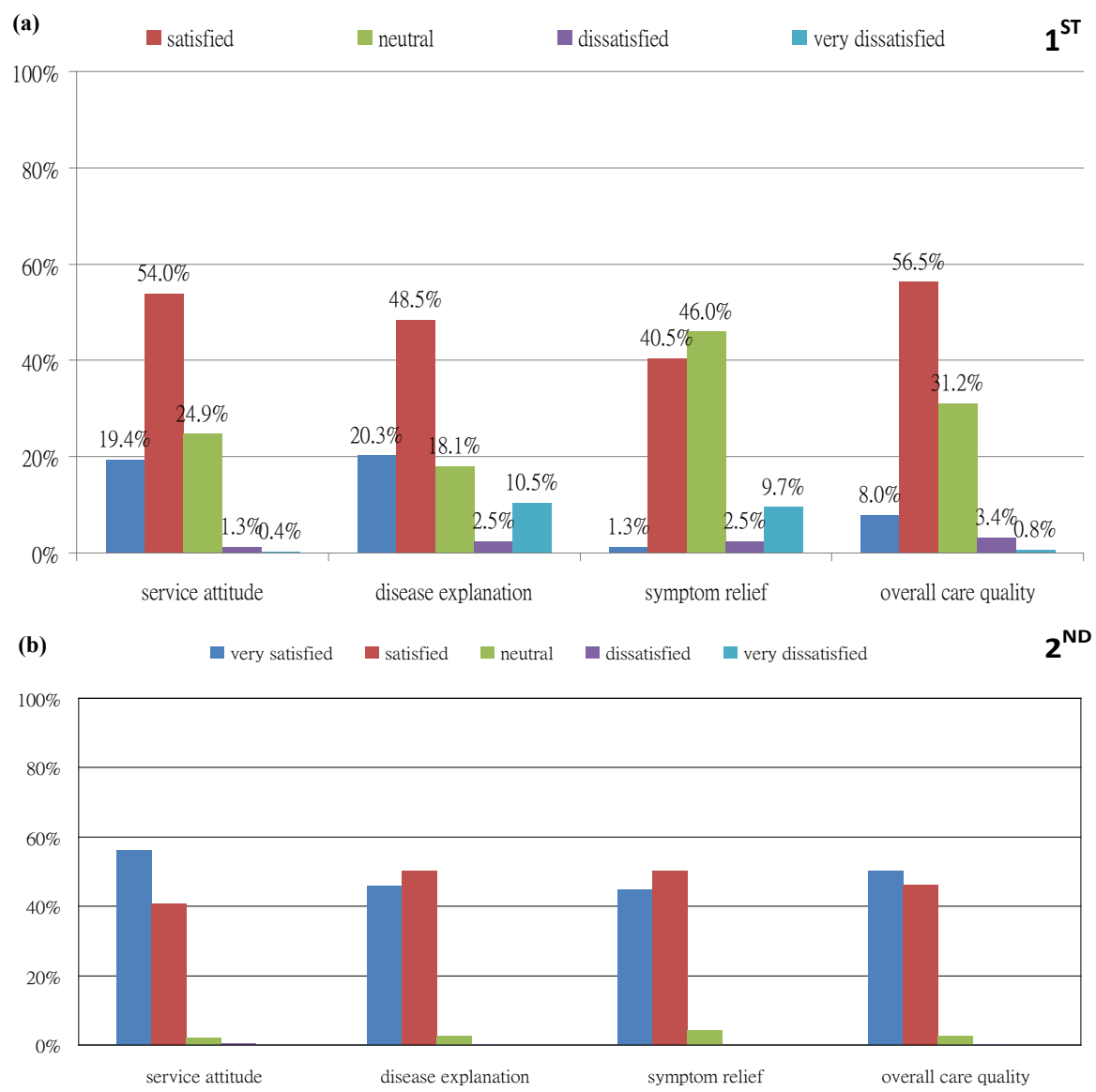

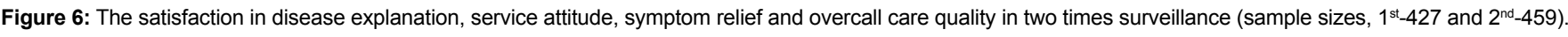

in both surveys with varied dimensions. The dissatisfaction was towards the long pre-hospitalized waiting time $(8 \%)$ and the lack of waiting space $(3.5 \%)$, that reminding us to do more efforts for improvement in the future.

\section{Advantages}

There was superiority of HOS care program at ED than setting at wards. Firstly, the working hours of hospitalists in HCU are reasonable, with 20 shifts and $8 \mathrm{~h}$ duty per shift every month $(160 \mathrm{~h} / \mathrm{month})$ to avoid burnouts, lessen stress and have more family relationships, which was different from physicians at wards who were responsible for all their hospitalized patients in full time. Secondly, through HCU team cooperation, time-consuming consultations were reduced which increased the efficiency of decision making and avoided redundant or repeat laboratory tests. Thirdly, collaboration between emergency physicians and hospitalists sharing medical knowledge, up-to-date guidelines and copying strategy in everyday handover meeting improved multidisciplinary thinking for whole-person care, it was held once a month in inter-team conference at wards. Fourthly, the severity of patient conditions was revealed by a color-coded system on computer screening (e.g. red=severe, yellow=variable, green=mild) to shorten the handover time and reminded physicians the disease status (color shifted by manipulation if patient conditions changed). Fifthly, the HCU team had the capacity for residency with interdisciplinary training about patient-centered but not a disease-oriented thinking. Sixthly, through experienced hospitalists care at ED, 55-60 patients were allowed discharge directly without hospitalization ( 18,333 USD/ per hospitalized day be saved). In general, HOS program at ED had above advantages than with this care model setting in hospitals at wards.

\section{Disadvantages}

There were some limitations in this program. Firstly, the complexity of co-morbidities and pre-hospitalized patient numbers were difficult to predict, and holistic care was time-consuming and sometimes physicians burden overloaded. Secondly, hospitalists were consisted of internal physicians and consultations for surgical conditions are usually made. Thirdly, this program was a bridging care between post-ED and prehospitalization, not likely setting at wards for total care until patient discharged. Fourthly, data came from only a medical center retrospectively, and no comparable setting at other hospitals. Regardless of advantages or disadvantages, this HOS program at ED ensured care quality and patient safety, which were directly cared by attending physicians. Moreover, it also saved medical expenditures, shortened length of stay and mortality at ED, and declined 6 and $24 \mathrm{~h}$ transferring to ICU after hospitalization. All of these enhanced the collaboration between hospitalists and emergency physicians to reduce burnouts or dismiss from ED to make a better working environment, to keep a quality of care and to create a multidisciplinary merit for pre-hospitalized patients.

\section{Conclusion}

The development of HOS model in Taiwan is challenging current healthcare system which was cared by residency as mentioned previously. Though HOS system had growing fast recently worldwide, 
Citation: Weng TC, Huang CC, Zhang CC, Huang HK, Wu MC, et al. (2017) Application of Integrated Medical Care Model (Hospitalist) in Emergency Department and its Efficiency and Outcome: 4 years' Experience in Taiwan. Health Care Current Reviews 5: 211. doi: 10.4172/23754273.1000211

it is just beginning in Taiwan and promoted with incentives by Ministry of Health and Welfare. From our 4 years experience, the utility of HOS program at ED earns a better patient care quality and also provides a multidisciplinary collaboration between physicians themselves. In recently, implementation of Noctunalist model at wards is launched in another medical center in Taiwan, to lessen the burdens of residency, was inspired by our HOS care model. Nevertheless, whether this model at ED have more efficiency and good result than setting at wards still warrants for further evaluation in future study. The HOS care model at ED is a pivotal approach in medical center, to our knowledge is also the first design in Taiwan. The 4 years outcome showed a decline in prehospitalized waiting time, over-waiting $(>48 \mathrm{~h}$ ) period, mortality rate, 6 and $24 \mathrm{~h}$ transferred to ICU after hospitalization, and saved medical costs, increased patient safety and satisfaction in care quality. It can be as reference to other hospitals in Taiwan having similar conditions of overcrowded patients at emergency room.

\section{Acknowledgement}

We thank Miss Yueh-Ling Huang, and Miss Richy Lee, for their helps in collecting data and statistics of SPSS version 20.

\section{References}

1. tma.tw/tma_ststs_2015/2015_stats.pdf

2. Wyatt R, Anderson-Drevs K, Van Male LM (2016) Workplace violence in health care: A critical issue with a promising solution. JAMA 316: 1037-1038.

3. Keller KL, Koenig WJ (1989) Sources of stress and satisfaction in emergency practice. J Emerg Med 7: 293-299.

4. Huang YC, Hsiao CT, Hsueh CC (2009) Suffering and expectation of patients waiting for ward boarding in the emergency department when hospitals are at full capacity. J Taiwan Emerg Med 11:109-116.

5. Wu TY, Majeed A, Kuo KN (2010) An overview of the healthcare system in Taiwan. Lond J Prim Care 3: 115-119.

6. Mowery NT, Dougherty SD, Hildreth AN, Holmes JH, Chang MC, et al. (2011) Emergency department length of stay is an independent predictor of hospital mortality in trauma activation patients. J Trauma 70: 1317-1325.

7. Chi-Mei Medical Center. Introduction.

8. Yang NP, Lee YH, Lin CH, Chung YC, Chen WJ, et al. (2009) Utilization of and direct expenditure for emergency medical care in Taiwan: A population-based descriptive study. J Epidemiol 19: 41-48.

9. Wachter RM, Goldman L (1996) The emerging role of "hospitalists" in the American health care system. NEJM 335: 514-517.

10. Messler J, Whitcomb WF (2015) A history of the hospitalist movement. Obstet Gynecol Clin North Am 42: 419-432.
11. López L, Hicks LS, Cohen AP, McKean S, Weissman JS (2009) Hospitalists and the quality of care in hospitals. Arch Intern Med 169: 1389-1394.

12. Peckham C (2016) Medscape hospitalist compensation report.

13. Lucena JF, Alegre F, Rodil R (2012) Results of a retrospective observationa study of intermediate care staffed by hospitalists: Impact on mortality comanagement, and teaching. J Hosp Med 7: 411-415

14. Roy C, Liang C, Lund M (2006) Implementation of a physician assistant hospitalist service in an academic medical center: Impact on efficiency and patient outcomes. J Hosp Med 3: 361-368.

15. Shu CC, Lin JW, Lin YF, Hsu NC, Ko WJ (2011) Evaluating the performance of a hospitalist system in Taiwan: A pioneer study for nationwide health insurance in Asia. J Hosp Med 6: 378-382.

16. Montgomery PW (2017) Hospitalists: the new wave of hospital medicine.

17. Meltzer D, Manning WG, Morrison J, Shah MN, Jin L, et al. (2002) Effects of physician experience on costs and outcomes on an academic general medicine service: Results of a trial of hospitalists. Ann Intern Med 137: 866-874.

18. Pines JM, Batt RJ, Hilton JA, Terwiesch C (2011) The financial consequences of lost demand and reducing boarding in hospital emergency departments. Ann Emerg Med 58: 331-340.

19. Rachoin JS, Skaf J, Cerceo E, Fitzpatrick E, Milcarek B, et al. (2012) The impact of hospitalists on length of stay and costs: Systematic review and metaanalysis. Am J Manag Care 18: e23-30.

20. Taiwan Ministry of Health and Welfare (2016) Hospitalists system.

21. Hsu NC. The hospitalists at wards of their outcomes (In Chinese). Reported of outcomes. Ministry of Health and Welfare.

22. Lin KC, Weng TC, Huang HK, Wu MC, Zhang CC, Hsu CC, et al. (2014) The improvement of emergency overcrowded: From hospitalist point of views. J Healthc Qual 8: 93-96.

23. Zhang CC, Lin KC, Chien TW (2017) Hospitalists in hospital emergency department help decrease the number of medical malpractices. Hospital 49 : $12-19$

24. Hung HK, Zhang CC, Weng TC, Yel CF, Liu TH, et al. (2016) Implementation of hospitalists at emergency room and the effects to diseases characteristic and overcrowding situations in Chi Mei Medical Center. Taiwan Med J 59:1-4.

25. Pollack CV Jr, Amin A, Talan DA (2012) Emergency medicine and hospital medicine: A call for collaboration. J Emerg Med 43: 328-334.

26. Shu CC, Hsu NC, Lin YF, Wang JY, Lin JW, et al. (2011) Integrated post discharge transitional care in a hospitalist system to improve discharge outcome: an experimental study BMC Med 9: 96.

27. Dedhia P, Kravet S, Bulger J, Hinson T, Sridharan A, et al. A quality improvemen intervention to facilitate the transition of older adults from three hospitals back to their homes. J Am Geriatr Soc 57: 1540-1546. 